\title{
Neutral current quasielastic (anti)neutrino scattering beyond the Fermi gas model at MiniBooNE and BNL kinematics
}

\author{
M. V. Ivanov* \\ Institute for Nuclear Research and Nuclear Energy, Bulgarian Academy of Sciences, Sofia 1784, Bulgaria \\ and Grupo de Física Nuclear, Departamento de Física Atómica, Molecular y Nuclear, \\ Facultad de Ciencias Físicas, Universidad Complutense de Madrid, E-28040 Madrid, Spain
}

A. N. Antonov

Institute for Nuclear Research and Nuclear Energy, Bulgarian Academy of Sciences, Sofia 1784, Bulgaria

M. B. Barbaro

Dipartimento di Fisica, Università di Torino, and INFN, Sezione di Torino, Via P. Giuria 1, I-10125 Torino, Italy

C. Giusti and A. Meucci

Dipartimento di Fisica, Università degli Studi di Pavia, and INFN, Sezione di Pavia, via Bassi 6, I-27100 Pavia, Italy

J. A. Caballero and R. González-Jiménez

Departamento de Física Atómica, Molecular y Nuclear, Universidad de Sevilla, 41080 Sevilla, Spain

E. Moya de Guerra and J. M. Udías

Grupo de Física Nuclear, Departamento de Física Atómica, Molecular y Nuclear, Facultad de Ciencias Físicas, Universidad Complutense de Madrid, Madrid E-28040, Spain

(Received 4 December 2014; revised manuscript received 26 January 2015; published 12 March 2015)

\begin{abstract}
Neutral current quasielastic (anti)neutrino scattering cross sections on a ${ }^{12} \mathrm{C}$ target are analyzed using a realistic spectral function $S(p, E)$ that gives a scaling function in accordance with the $\left(e, e^{\prime}\right)$ scattering data. The spectral function accounts for the nucleon-nucleon $(\mathrm{NN})$ correlations by using natural orbitals from the Jastrow correlation method and has a realistic energy dependence. The standard value of the axial mass $M_{A}=1.032 \mathrm{GeV}$ is used in all calculations. The effect of the final-state interaction on the spectral and scaling functions, as well as on the cross sections, is accounted for. A comparison of the calculations with the empirical data of the MiniBooNE and BNL experiments is performed. Our results are analyzed in comparison with those when NN correlations are not included and, also, with results from other theoretical approaches, such as the relativistic Fermi gas, the relativistic mean field, and the relativistic Green's function, as well as with the SuperScaling Approach based on the analysis of quasielastic electron scattering.
\end{abstract}

DOI: 10.1103/PhysRevC.91.034607

PACS number(s): 25.30.Pt, 13.15.+g, 24.10.Jv

\section{INTRODUCTION}

The analyses of $y$ scaling (see, e.g., [1-10]) and superscaling (based on the $\psi^{\prime}$-scaling variable) (see, e.g., [10-21]) phenomena in inclusive electron scattering on nuclei have induced studies of (anti)neutrino-nucleus scattering on the same basis. This allows one to explore fundamental questions of neutrino reactions and neutrino oscillations in relation to the hypothesis of nonzero neutrino masses [22]. The theoretical concept of superscaling (a very weak dependence of the reduced cross section on the momentum transfer $q$ at excitation energies below the quasielastic $(\mathrm{QE})$ peak for large enough $q$ and no dependence on the mass number) has been introduced $[10,11]$ within the relativistic Fermi gas (RFG) model. It has been pointed out in [13], however, that the actual dynamical reason for superscaling is more complex than that provided by the RFG model. This imposes the necessity to consider

*martin.inrne@gmail.com superscaling in the framework of theoretical methods that go beyond the RFG model. An example is the coherent density fluctuation model [23,24], used in [16-19] and [25] within this context.

In [26] the analyses of superscaling have been extended to include not only $\mathrm{QE}$ processes but also those in which $\Delta$ excitation dominates. The $\mathrm{QE}$ and $\Delta$-region scaling functions $f^{\mathrm{QE}}\left(\psi^{\prime}\right)$ and $f^{\Delta}\left(\psi^{\prime}\right)$ have been deduced in [26] from phenomenological fits to the data for electron-nucleus scattering cross sections by dividing the latter by the appropriate elementary $N \rightarrow N$ and $N \rightarrow \Delta$ functions, respectively. Therefore they include all the effects of the nuclear dynamics, in particular, nucleon-nucleon (NN) correlations and final-state interactions (FSIs), which should be reproduced by reliable nuclear models. For instance, in [27] and [28] a QE scaling function with an asymmetric shape has been obtained, in agreement with the experimental scaling function using the relativistic mean-field (RMF) model for the final states.

In order to exploit superscaling for neutrino-nucleus studies, in [26] (see also [27] and [29]) the above procedure 
has been inverted: the scaling functions have been multiplied by the elementary charged current (CC) (anti)neutrino cross section to obtain the corresponding CC (anti)neutrino cross sections on nuclei for intermediate to high energies in the same region of excitation. The scaling and superscaling ideas have been carried a step further in [30] to include neutral current (NC) (anti)neutrino scattering cross sections from ${ }^{12} \mathrm{C}$, namely, the reactions ${ }^{12} \mathrm{C}(\nu, p v) \mathrm{X}$ and ${ }^{12} \mathrm{C}(\bar{v}, p \bar{v}) \mathrm{X}$, involving proton knockout, and ${ }^{12} \mathrm{C}(\nu, n \nu) \mathrm{X}$ and ${ }^{12} \mathrm{C}(\bar{\nu}, n \bar{v}) \mathrm{X}$, involving neutron knockout in the $\mathrm{QE}$ regime. The coherent density fluctuation model scaling function was applied to analyses of NC (anti)neutrino scattering on ${ }^{12} \mathrm{C}$ with energies of $1 \mathrm{GeV}$ ( $u$-channel-inclusive processes) in [25]. A number of other theoretical studies have been devoted to both NC (e.g., [3134]) and CC (e.g., [32-41]) neutrino scattering on nuclei.

Our interest in the present work concerns the analysis of different experimental data recently obtained on neutrinonucleus processes at several facilities. At around $1 \mathrm{GeV}$, data are available from the MiniBooNE Collaboration, for both $\mathrm{CC}$ [42] and $\mathrm{NC}$ [43] neutrino- ${ }^{12} \mathrm{C}$ processes, and previous experiment [44]. We note also that recent data on antineutrinonucleus scattering are reported in [45] and [46]. It is known that analyses of nuclear effects in neutrino scattering are generally regarded as one of the main sources of systematic uncertainties in oscillation experiments, in particular, when it concerns the understanding of charged current/quasielastic (CCQE) interactions with nucleons bound in the nucleus (e.g., [47]) in the energy range of around $1 \mathrm{GeV}$. For energies of a few $\mathrm{GeV}$, the $\Delta$-resonance excitation becomes equally important [48] (but keep in mind that here the nuclear uncertainties are even larger).

Though the main subject of our work is neutral current/quasielastic (NCQE) neutrino scattering by nuclei, in what follows we note various theoretical models that have also been used for the description of CC processes in connection with their applications to analyses of NC processes.

Analyses of the CCQE MiniBooNE data have raised questions about the capabilities of the various models to account for different contributions to the neutrino-nucleus scattering cross sections. The RFG model, in which the shell structure and the nucleon correlations are neglected, gives results for CCQE neutrino scattering that underestimate the data. Accordance with the data is achieved by increasing the world-average axial mass $M_{A}\left(M_{A}=1.032 \mathrm{GeV}\right)$ to $M_{A}=1.35 \mathrm{GeV}$. An enhancement of the world-average axial mass is required also in other models based on the impulse approximation (e.g., [47] and [49-56]). This is an indication that models based on the impulse approximation may lack important contributions to the processes of neutrino-nucleus scattering. In approaches beyond the impulse approximation, ingredients such as two-particle two-hole $(2 p-2 h)$ contributions have been included. In Refs. [57] and [58] an approach based on the random-phase approximation, improved by considering relativistic corrections [52], led to a good agreement with the MiniBooNE data for both CCQE and NCQE scattering, including the double-differential CCQE cross section. It was pointed out in [53] that the multinucleon contribution may effectively be accounted for by increasing the value of the axial mass. As shown in [54] and [59-61], the RMF approach gives a good description of the shape of the double-differential cross section from the MiniBooNE experiment but fails to reproduce its normalization. It has been noted in [54] that meson exchange currents (MEC) could reduce the discrepancy. The calculations within the relativistic Green's function (RGF) model $[49,62,63]$ have provided a good description of the total CCQE and of the (double-) differential (CCQE) NCQE MiniBooNE cross sections [49,55,61,62,64]. The larger RGF cross sections can be attributed to the overall effect of inelastic channels, for instance, rescattering of the knockedout nucleon, multinucleon processes, and non-nucleonic $\Delta$ excitation, that are recovered in the model by the use of a complex relativistic optical potential to describe FSIs and that are not included in other models based on the impulse approximation.

The SuperScaling Approach (SuSA) previously discussed has been used for analyses of neutrino-nucleus processes in numerous studies $[26,30,54,59,65,66]$. The results of the SuSA underestimate the MiniBoonE data by $20 \%-30 \%$. An updated version of the model (SuSAv2), which incorporates different $\mathrm{RMF}$ effects in the longitudinal and transverse channels as well as isospin dependence, yields a milder disagreement (10\%-15\%) [66]. The account for MEC significantly increases the results for the cross sections, reducing the discrepancy with the data in the antineutrino case but not so much in the neutrino case.

Multinucleon effects on $\mathrm{CC}$ neutrino-nucleus scattering have also been investigated by the use of the Giessen Boltzmann-Uehling-Uhlenback event generator in Ref. [67], where the energy spectra of the knockout nucleons are given in detail. In parallel, it is also interesting to compare with the older NCQE data from the Brookhaven National Laboratory (BNL) E734 experiment [44], corresponding to neutrino kinematics similar to MiniBooNE. We should emphasize that the NC cross sections depend also on the strangeness content of the nucleon, particularly through the axial form factor. Hence good control of nuclear effects is also required if one wants to extract information on the strange form factors of the nucleon from $\mathrm{NC}$ data.

In [47] the differential and total cross sections for energies ranging from a few hundred $\mathrm{MeV}$ to $100 \mathrm{GeV}$ have been obtained and compared with the data from BNL E734, MiniBooNE, and NOMAD (see Ref. [68]). It is concluded in [47] that the nuclear effects in NCQE and CCQE scattering seem to be very similar, though, according to the authors, combined analyses of the CCQE and NCQE data do not seem to support the contribution of multinucleon final states being large enough to explain the normalization of the MiniBooNE cross sections. It should be mentioned that in [47] an effective value of $M_{A}=1.23 \mathrm{GeV}$ is used.

The sensitivity to FSIs of NCQE (anti)neutrino scattering cross sections has been investigated in [62], where RGF cross sections calculated with different parametrizations for the phenomenological relativistic optical potential are compared with MiniBooNE data. The RGF results obtained with the standard value of the axial mass describe well the NCQE (anti)neutrino scattering data. It is pointed out, however, that application of the RGF model to the semi-inclusive NCQE scattering can also include contributions of channels which 
are present in the inclusive but not in the semi-inclusive reaction.

More theoretical approaches are presented in Ref. [50], where an axial mass $M_{A}=1.28 \pm 0.05 \mathrm{GeV}$ and a strangeness $\Delta s=0.11 \pm 0.36$ are extracted, and in Ref. [69] within the transverse enhancement model [70,71], where a good agreement between the theoretical results and the NCQE data is obtained, that the authors interpret as a very sizable contribution of $2 p-2 h$ and $3 p-3 h$ processes. Finally, in Ref. [72] an approach based on a realistic spectral function was applied to calculate the neutron-knockout (through NC scattering) cross sections in the kinematical regime of atmospheric-neutrino interactions in a broad energy range extending to $10 \mathrm{GeV}$.

The main aim of this paper is to analyze NCQE (anti)neutrino scattering cross sections on a ${ }^{12} \mathrm{C}$ target using a realistic spectral function, $S(p, \mathcal{E})$, that gives a scaling function in accordance with the $\left(e, e^{\prime}\right)$ scattering data. In our previous work [73] this approach was applied to calculate CCQE (anti)neutrino scattering on ${ }^{12} \mathrm{C}$. The spectral function accounts for the NN correlations by using natural orbitals (NOs) from the Jastrow correlation method and has a realistic energy dependence. In the calculations the standard value of the axial mass $M_{A}=1.032 \mathrm{GeV}$ is used. As in [73], in the present work the effect of FSIs on the scaling functions and on the cross sections is taken into account. A comparison of the calculations with the empirical data from the MiniBooNE and BNL experiments is performed.

The theoretical scheme of the work is given in Sec. II, where the method for obtaining a realistic spectral function, as well as the main relationships concerning the NCQE (anti)neutrino-nucleus reaction cross sections, is presented. The results of the calculations and the discussion are given in Sec. III, while a summary of the work and the conclusions are included in Sec. IV.

\section{THEORETICAL SCHEME}

The general formalism for NC (anti)neutrino scattering in the $\mathrm{QE}$ regime has been introduced in many previous works [30,33,74-77]. Here we summarize briefly those aspects which are of more relevance for the later discussion of the results and of their comparison with MiniBooNE and BNL data. We consider the semileptonic quasifree scattering from nuclei in the Born approximation, assuming that the inclusive cross sections are well represented by the sum of the integrated semiinclusive proton and neutron emission cross sections [30]. The kinematics for semileptonic nucleon knockout reactions in the one-boson-exchange approximation is presented in Fig. 1.

A lepton with 4-momentum $K^{\mu}=(\epsilon, \mathbf{k})$ scatters to another lepton with 4-momentum $K^{\prime \mu}=\left(\epsilon^{\prime}, \mathbf{k}^{\prime}\right)$, exchanging a vector boson with 4-momentum $Q^{\mu}=K^{\mu}-K^{\prime \mu}$. The lepton energies are $\epsilon=\sqrt{m^{2}+k^{2}}$ and $\epsilon^{\prime}=\sqrt{m^{\prime 2}+k^{\prime 2}}$, where the masses of the initial and final lepton $m$ and $m^{\prime}$ are assumed to be equal to 0 for $\mathrm{NC}$ neutrino scattering. In the laboratory system the initial nucleus, being in its ground state, has a 4-momentum $P_{A}^{\mu}=\left(M_{A}^{0}, 0\right)$, while the final hadronic state corresponds to a proton or neutron with 4-momentum $P_{N=p \text { or } n}^{\mu}=\left(E_{N}, \mathbf{p}_{N}\right)$ and an unobserved residual nucleus with 4-momentum $P_{B}^{\mu}=$ $\left(E_{B}, \mathbf{p}_{B}\right)$. Usually the missing momentum $\mathbf{p} \equiv-\mathbf{p}_{B}$ and the

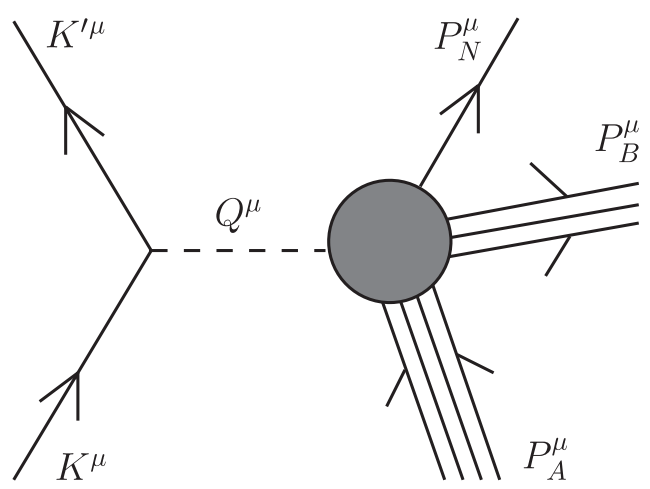

FIG. 1. Kinematics for semileptonic nucleon knockout reactions in the one-boson-exchange approximation.

excitation energy $\mathcal{E} \equiv E_{B}-E_{B}^{0}$, with $E_{B}^{0}=\sqrt{\left(M_{B}^{0}\right)^{2}+p^{2}}$, are introduced, $M_{B}^{0}$ being the ground-state mass of the daughter nucleus. Although in real situations, as is the case for MiniBooNE and BNL, there are usually no monochromatic beams and an integral over the allowed energies folded with the neutrino flux must be performed, we assume the energy of the incident neutrino to be specified and also the outgoing nucleon energy $E_{N}$ to be known. Finally, the angle $\theta_{k p_{N}}$ between the incident neutrino and the ejected nucleon momentum is also given.

Starting from the Feynman amplitude associated with the exclusive diagram in Fig. 1, one can get inclusive cross sections by integrating over the undetected outgoing particles.

In the case of QE electron or CCQE neutrino scattering, the outgoing lepton is detected and a sum over the outgoing nucleon variables is performed. Using the language introduced in this context in Ref. [74], we refer to these processes as " $t$ channel" reactions, since the Mandelstam variable $t=\left(K^{\mu}-\right.$ $\left.K^{\prime \mu}\right)^{2}$ is fixed. Then the $\left(e, e^{\prime}\right)$ and $\mathrm{CC}\left(v_{l}, l^{\prime}\right)$ reactions are governed by the same kinematics and the scaling formalism developed for the former can be trivially extended to the latter.

In the case of $\mathrm{NC}$ neutrino scattering, only the outgoing nucleon can be experimentally detected, while the unobserved outgoing neutrino is integrated over. This is referred to as a " $u$-channel" process, where the Mandelstam variable $u=$ $\left(K^{\mu}-P^{\mu}\right)^{2}$ is fixed. Then the kinematics is not the same as in the $\left(e, e^{\prime}\right)$ case, and in particular, the two inclusive cross sections involve an integration over a slightly different region in the missing energy and momentum plane. As a consequence, it is not evident that the scaling arguments can be applied to $\mathrm{NC}$ scattering. However, in [30] the influence of a nonconstant $Q^{\mu}$ in the derivation of scaling in the NC case was thoroughly investigated within the general framework of the RFG model and it was concluded that the scaling ideas still work properly for NC neutrino-nucleus processes. That study was extended in [77] and [78] making use of the RMF approach. These results showed that scaling of the second kind, i.e., independent of the nuclear target, works extremely well. On the contrary, scaling of the first kind (independent of the transfer momentum) depends on the specific kinematical situation considered. In general, first-kind scaling seems to be well respected when the angle of the ejected nucleon is larger than roughly $50^{\circ}$. 
This is the region where the cross section integrated over angles reaches larger values. Therefore, first-kind scaling is expected to work properly at MiniBooNE and BNL. Indeed, for the RMF and the particular kinematics involved in the experiments analyzed in this work (MiniBooNE and BNL), we have verified that calculation of the NC cross sections based on $u$ scaling [as indicated in Eqs. (10) and (11) plus (8)] gives rise to results very similar (within a few percent) to those provided by the full calculation, i.e., without resorting to the scaling assumption. This is strictly true for $Q^{2} \lesssim 0.7 \mathrm{GeV}^{2}$. For larger values of $Q^{2}$, the $u$-scaling approximation begins to deviate from the full result, but by an amount significantly smaller than the uncertainty linked to the data error bands.

The usual procedure for calculating the $\left(l, l^{\prime} N\right)$ cross section includes the plane-wave impulse approximation (PWIA) and and integrations over all unconstrained kinematic variables. It is shown in [30] that the inclusive cross section in the $u$ channel can be written, after some approximations, in the form

$$
\frac{d \sigma}{d \Omega_{N} d p_{N}} \simeq \bar{\sigma}_{\text {s.n. }}^{(u)} F\left(y^{\prime}, q^{\prime}\right),
$$

where

$$
F\left(y^{\prime}, q^{\prime}\right) \equiv \int_{\mathcal{D}_{u}} p d p \int \frac{d \mathcal{E}}{E} \Sigma \simeq F\left(y^{\prime}\right),
$$

provided the effective NC single-nucleon (s.n.) cross section

$$
\begin{aligned}
\bar{\sigma}_{\text {s.n. }}^{(u)}= & \frac{1}{32 \pi \epsilon} \frac{1}{q^{\prime}}\left(\frac{p_{N}^{2}}{E_{N}}\right) g^{4} \int_{0}^{2 \pi} \frac{d \phi^{\prime}}{2 \pi} l_{\mu \nu}\left(\mathbf{k}, \mathbf{k}^{\prime}\right) \\
& \times w^{\mu \nu}\left(\mathbf{p}, \mathbf{p}_{N}\right) D_{V}\left(Q^{2}\right)^{2}
\end{aligned}
$$

is almost independent of $(p, \mathcal{E})$ for constant $\left(k, p_{N}, \theta_{k p_{N}}\right)$. In Eq. (3) $l_{\mu \nu}$ and $w^{\mu \nu}$ are the leptonic and s.n. hadronic tensor, respectively, and $D_{V}\left(Q^{2}\right)$ is the vector boson propagator [30]. In Eq. (1) $Q^{\prime \mu} \equiv K^{\mu}-P_{N}^{\mu}=\left(\omega^{\prime}, \mathbf{q}^{\prime}\right)$ is the 4-momentum transferred from the initial lepton to the ejected nucleon and $y^{\prime}$ is the scaling variable naturally arising in the $u$-scattering kinematics, analogous to the usual $y$-scaling variable for $t$ scattering. The scaling function $F\left(y^{\prime}\right)$ obtained within a given approach can be used to predict realistic NC cross sections. Assuming that the domains of integration $\mathcal{D}_{u}$ (in the $u$ channel) and $\mathcal{D}_{t}$ (in the $t$ channel) are the same or very similar, the results for the scaling function obtained in the case of inclusive electron scattering (where $\mathcal{D}_{t}$ works) can be used in the case of NC neutrino reactions. It is pointed out in [30] that $\mathcal{D}_{t}$ and $\mathcal{D}_{u}$ differ significantly only at large $\mathcal{E}$ (also at large $p$, but there the semi-inclusive cross sections are expected to be negligible). So, given that the semi-inclusive cross sections are dominated by their behavior at low $\mathcal{E}$ and low $p$, one expects the results of the integrations in the $t$ and $u$ channel to be very similar, and thus the scaling functions will be essentially the same in both cases.

As noted in [30], if the s.n. cross section is smoothly varying within the $(p, \mathcal{E})$ integration region, the differential cross section in the RFG can be factorized as shown in Eq. (1) with the RFG scaling function,

$$
F_{\mathrm{RFG}}\left(\psi_{\mathrm{RFG}}\right)=\frac{3}{4} k_{F}\left(1-\psi_{\mathrm{RFG}}^{2}\right) \theta\left(1-\psi_{\mathrm{RFG}}^{2}\right),
$$

where the RFG $u$-channel $\psi$ variable is defined as

$$
\psi_{\mathrm{RFG}}=s \sqrt{\frac{m_{N}}{T_{F}}}\left[\sqrt{1+\left(\frac{y_{\mathrm{RFG}}}{m_{N}}\right)^{2}}-1\right]^{1 / 2}
$$

and

$$
y_{\mathrm{RFG}}=s \frac{m_{N}}{\tau^{\prime}}\left[\lambda^{\prime} \sqrt{\tau^{\prime 2} \rho^{\prime 2}+\tau^{\prime}}-\kappa^{\prime} \tau^{\prime} \rho^{\prime}\right]
$$

is the RFG $y$-scaling variable for the $u$ channel and corresponds to the minimum momentum required for a nucleon to participate in NC neutrino-nucleus scattering. The dimensionless kinematic quantities in Eq. (6) are given by $\kappa^{\prime} \equiv q^{\prime} / 2 m_{N}$, $\lambda^{\prime} \equiv \omega^{\prime} / 2 m_{N}, \tau^{\prime}=\kappa^{\prime 2}-\lambda^{\prime 2}$, and $\rho^{\prime} \equiv 1-\frac{1}{4 \tau^{\prime}}\left(1-m^{\prime 2} / m_{N}^{2}\right)$. The sign $s$ is

$$
s \equiv \operatorname{sgn}\left\{\frac{1}{\tau^{\prime}}\left[\lambda^{\prime} \sqrt{\tau^{\prime 2} \rho^{\prime 2}+\tau^{\prime}}-\kappa^{\prime} \tau^{\prime} \rho^{\prime}\right]\right\} .
$$

The basic relationships used to calculate the s.n. cross sections are given in [30]. This concerns the leptonic and hadronic tensors and the response and structure functions. Here we summarize the basic expressions for the neutral weak nucleon form factors and the specific set of parameters considered in the calculations:

(i) The weak mixing angle $\sin ^{2} \theta_{W}=0.23122(15)$ [79].

(ii) The isovector axial form factor of the nucleon at zero momentum transfer $g_{A}=1.2695$ [79].

(iii) The weak form factors are given in the form (Eqs. (55) and (56), Ref. [80])

$$
\begin{aligned}
\widetilde{G}_{a}\left(Q^{2}\right) & =\xi_{V}^{T=1} G_{a}^{T=1} \tau_{3}+\sqrt{3} \xi_{V}^{T=0} G_{a}^{T=0}+\xi_{V}^{(0)} G_{a}^{(s)}, \\
a & =\{E, M\}, \\
\widetilde{G}_{A}\left(Q^{2}\right) & =\xi_{A}^{T=1} G_{A}^{(3)} \tau_{3}+\xi_{A}^{T=0} G_{A}^{(8)}+\xi_{A}^{(0)} G_{A}^{(s)},
\end{aligned}
$$

with (no radiative corrections included)

$$
\begin{gathered}
\xi_{V}^{T=1}=2\left(1-2 \sin ^{2} \theta_{W}\right), \quad \sqrt{3} \xi_{V}^{T=0}=-4 \sin ^{2} \theta_{W}, \\
\xi_{V}^{(0)}=-1, \quad \xi_{A}^{T=1}=-2, \quad \xi_{A}^{T=0}=0, \quad \xi_{A}^{(0)}=1 .
\end{gathered}
$$

(iv) The isoscalar and isovector form factors are (for more details, see [80])

$$
\begin{aligned}
G_{E, M}^{T=1} & =G_{E, M}^{p}-G_{E, M}^{n}, \quad G_{E, M}^{T=0}=G_{E, M}^{p}+G_{E, M}^{n}, \\
G_{A}^{(3)} & =g_{A} / 2 G_{D}^{A}\left(Q^{2}\right), \quad G_{A}^{(s)}=g_{A}^{(s)} G_{D}^{A}\left(Q^{2}\right),
\end{aligned}
$$

with $G_{D}^{A}\left(Q^{2}\right)=\left(1+\left|Q^{2}\right| / M_{A}^{2}\right)^{-2}$.

(v) Electric and magnetic strange form factors (dipole $Q^{2}$ dependence has been used)

$$
G_{E}^{(s)}\left(Q^{2}\right)=\rho_{s} \tau G_{D}^{V}\left(Q^{2}\right), \quad G_{M}^{(s)}\left(Q^{2}\right)=\mu_{s} G_{D}^{V}\left(Q^{2}\right) .
$$

(vi) The electromagnetic form factors correspond to the so-called GKex model (Gari-Krümpelmann extended, developed by E. L. Lomon) [81-83].

(vii) Unless otherwise specified, the following set of strange parameters has been considered (see Ref. [80] for more details):

$$
\mu_{s}=-0.020 ; \rho_{s}=0.59 ; g_{A}^{(s)}=0 .
$$


(viii) Finally, the axial mass is taken to be the worldaverage value $M_{A}=1.032 \mathrm{GeV}$.

In this work we use in the calculations the RFG, SuSA, harmonic oscillator $(\mathrm{HO}+\mathrm{FSI})$ and NOs (NO + FSI) scaling functions including FSIs (see [73] and the text below). We present also results obtained in the RMF and RGF models, where the cross sections are calculated in a fully unfactorized approach which does not make use of the approximations leading to Eq. (1). All our results are used to analyze NCQE (anti)neutrino cross sections on a $\mathrm{CH}_{2}$ target measured by the MiniBooNE Collaboration [43,46]. We also make comparisons with the BNL E734 experiment [44], studying $v p$ and $\bar{v} p$ NCQE interactions, where the target was composed in $79 \%$ of protons bound in carbon and aluminum and in $21 \%$ of free protons.

In detail, here we present briefly how the HO + FSI and $\mathrm{NO}+$ FSI scaling functions are obtained (see also Ref. [73]):

(i) The spectral function $S(p, \mathcal{E})$ is constructed in the form

$$
S(p, \mathcal{E})=\sum_{i} 2\left(2 j_{i}+1\right) n_{i}(p) L_{\Gamma_{i}}\left(\mathcal{E}-\mathcal{E}_{i}\right) .
$$

(ii) The single-particle momentum distributions $n_{i}(p)$ are taken to correspond either to the HO single-particle wave functions or to the NOs from the Jastrow correlation method, where central short-range $\mathrm{NN}$ correlations are included.

(iii) The Lorentzian function,

$$
L_{\Gamma_{i}}\left(\mathcal{E}-\mathcal{E}_{i}\right)=\frac{1}{\pi} \frac{\Gamma_{i} / 2}{\left(\mathcal{E}-\mathcal{E}_{i}\right)^{2}+\left(\Gamma_{i} / 2\right)^{2}},
$$

is used for the energy dependence of the spectral function with parameters $\Gamma_{1 p}=6 \mathrm{MeV}$ and $\Gamma_{1 s}=$ $20 \mathrm{MeV}$, which are fixed to the experimental widths of the $1 p$ and $1 s$ states in ${ }^{12} \mathrm{C}$.

The realistic spectral function $S(p, \mathcal{E})$ is presented in Fig. 2, where the two shells $1 p$ and $1 s$ are clearly visible.

(iv) For a given momentum transfer $q$ and energy of the initial electron $\varepsilon$ we calculate the electron-nucleus

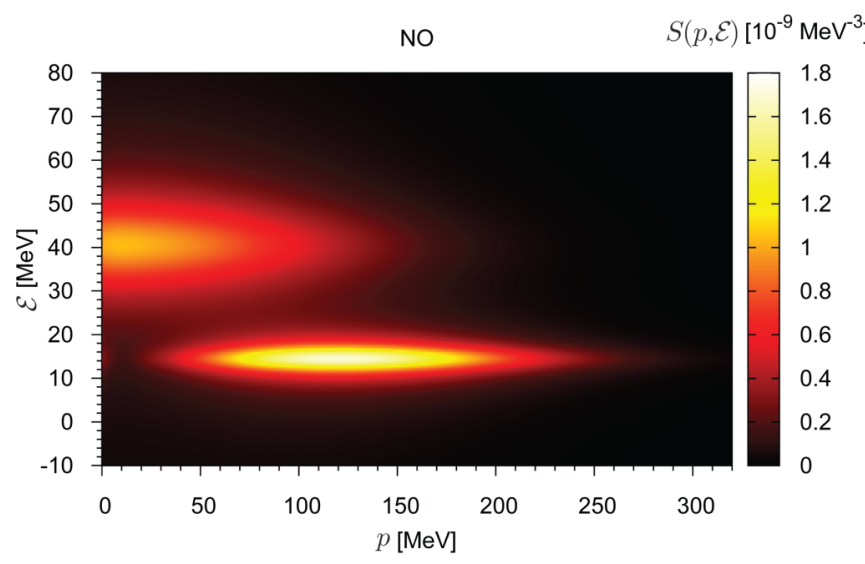

FIG. 2. (Color online) The ${ }^{12} \mathrm{C}$ realistic spectral function $S(p, \mathcal{E})$, which is constructed using natural orbit single-particle momentum distributions from the Jastrow correlation method and Lorentzian function for the energy dependence (see text). $\left({ }^{12} \mathrm{C}\right)$ cross section using

$$
\begin{aligned}
\frac{d \sigma_{t}}{d \omega d|\mathbf{q}|}= & 2 \pi \alpha^{2} \frac{|\mathbf{q}|}{E_{\mathbf{k}}^{2}} \int d E d^{3} p \frac{S_{t}(\mathbf{p}, E)}{E_{\mathbf{p}} E_{\mathbf{p}^{\prime}}} \\
& \times \delta\left(\omega+M-E-E_{\mathbf{p}^{\prime}}\right) L_{\mu \nu}^{\mathrm{em}} H_{\mathrm{em}, t}^{\mu \nu},
\end{aligned}
$$

in which the spectral function $S(p, \mathcal{E})$ [Eq. (8)] is used, the index $t$ denotes the nucleon isospin, and $L_{\mu \nu}^{\mathrm{em}}$ and $H_{\mathrm{em}, t}^{\mu \nu}$ are the leptonic and hadronic tensors, respectively (for details, see Ref. [73]).

(v) The corresponding scaling function $F(q, \omega)$ is calculated within the PWIA by

$$
F(q, \omega) \cong \frac{\left[d \sigma / d \epsilon^{\prime} d \Omega^{\prime}\right]_{\left(e, e^{\prime}\right)}}{\bar{\sigma}^{e N}(q, \omega ; p=|y|, \mathcal{E}=0)},
$$

and by multiplying it by $k_{F}$ the scaling function $f(\psi)$ is obtained, where $k_{F}$ is the Fermi momentum for a specific nucleus and $\bar{\sigma}^{e N}$ is the azimuthal angleaveraged s.n. cross section.

(vi) To account for FSIs, the $\delta$ function in Eq. (10) is replaced by

$$
\begin{aligned}
& \delta\left(\omega+M-E-E_{\mathbf{p}^{\prime}}\right) \\
& \quad \rightarrow \frac{W / \pi}{W^{2}+\left[\omega+M-E-E_{\mathbf{p}^{\prime}}-V\right]^{2}},
\end{aligned}
$$

where the real $(V)$ and imaginary $(W)$ parts of the optical potential are obtained in Ref. [84] from the Dirac optical potential.

\section{RESULTS AND DISCUSSION}

In this section the theoretical predictions of the RFG, $\mathrm{HO}+\mathrm{FSI}, \mathrm{NO}+\mathrm{FSI}$, and SUSA scaling functions are compared with the data measured by the MiniBooNE and BNL collaborations. The comparison is performed also with the results of the RMF and RGF models, which are based on the same RMF model for nuclear structure but on a different treatment of FSIs. In the RMF model FSIs are described by the same RMF potential describing the initial nucleon state; the description of FSIs in the RGF is based on the use of a complex optical potential. Details of the RGF model can be found, for instance, in [85] and [86]. The results of the RMF and RGF models have been widely compared in [63] for inclusive QE electron scattering, in [55] and [87] for CCQE, and in [61] for NCQE neutrino scattering. RGF calculations presented in this work were carried out with the recent democratic optical potential of [88] (RGF-DEM).

The comparison between theory and experiment for the NCQE flux-averaged MiniBooNE (anti)neutrino cross section is presented in Fig. 3. Here we compare the predictions using the RFG, $\mathrm{HO}+$ FSI, NO + FSI, and SUSA scaling functions and the RMF model with the data. As usual in NC reactions, in this work, the variable $Q^{2}$ is defined as $Q^{2}=$ $2 M_{N} T_{N}$, where $M_{N}$ and $T_{N}$ are the mass and kinetic energy of the outgoing nucleon, respectively. In order to compare with MiniBooNE we evaluate the following differential cross 

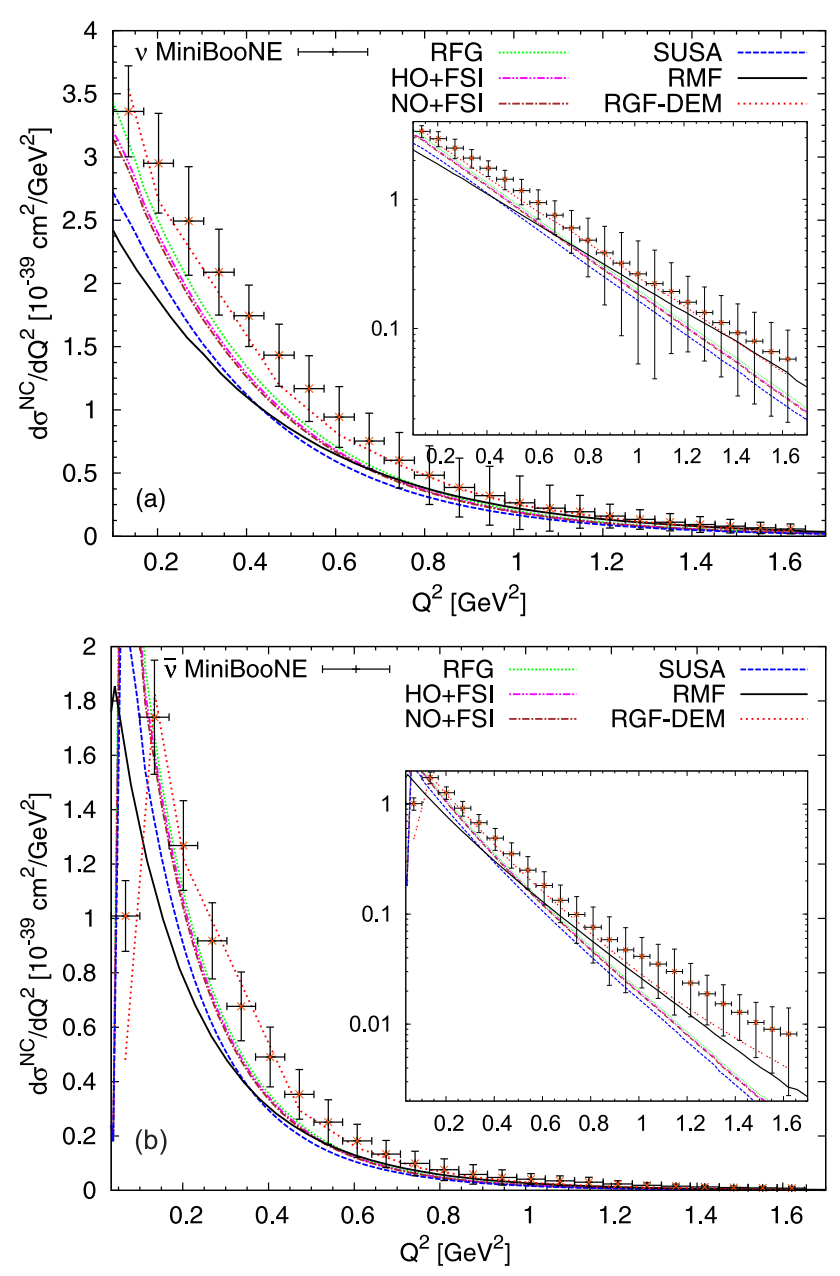

FIG. 3. (Color online) NCQE neutrino [(a) $v N \rightarrow v N]$ and antineutrino [(b) $\bar{v} N \rightarrow \bar{v} N$ ] flux-averaged differential cross section computed using the RFG, HO + FSI, NO + FSI, and SUSA scaling functions in the RGF and RMF models and compared with the MiniBooNE data $[43,46]$. Results correspond to the world-average axial mass $M_{A}=1.032 \mathrm{GeV}$ and strangeness $\Delta s=0$. Error bars do not account for the normalization uncertainty of $18.1 \%(19.5 \%)$ in the $v(\bar{v})$ case.

section per nucleon,

$$
\begin{aligned}
\frac{d \sigma_{v(\bar{v}) N \rightarrow v(\bar{v}) N}}{d Q^{2}}= & \frac{1}{7} C_{v(\bar{v}) p, \mathrm{H}}\left(Q^{2}\right) \frac{d \sigma_{v(\bar{v}) p \rightarrow v(\bar{v}) p, \mathrm{H}}}{d Q^{2}} \\
& +\frac{3}{7} C_{v(\bar{v}) p, \mathrm{C}}\left(Q^{2}\right) \frac{d \sigma_{v(\bar{v}) p \rightarrow v(\bar{v}) p, \mathrm{C}}}{d Q^{2}} \\
& +\frac{3}{7} C_{v(\bar{v}) n, \mathrm{C}}\left(Q^{2}\right) \frac{d \sigma_{v(\bar{v}) n \rightarrow v(\bar{v}) n, \mathrm{C}}}{d Q^{2}},
\end{aligned}
$$

where $C_{v(\bar{v}) p, \mathrm{H}}\left(Q^{2}\right), C_{v(\bar{v}) p, \mathrm{C}}\left(Q^{2}\right)$, and $C_{v(\overline{\mathrm{v}}) n, \mathrm{C}}\left(Q^{2}\right)$ are the efficiency correction functions, given in Refs. [43] and [46], of three processes: the (anti)neutrino scattering off free protons in the hydrogen atom, the bound protons in the carbon atom, and the bound neutrons in the carbon atom. $d \sigma_{v(\bar{v})} p \rightarrow v(\bar{v}) p, \mathrm{H} / d Q^{2}$, $d \sigma_{v(\bar{v}) p \rightarrow v(\bar{v}) p, \mathrm{C}} / d Q^{2}$, and $d \sigma_{v(\bar{v}) n \rightarrow v(\bar{v}) n, \mathrm{C}} / d Q^{2}$ are the theoretical $v(\bar{v})$ NCQE cross sections on free protons (per free proton), on bound protons (per bound proton), and on bound

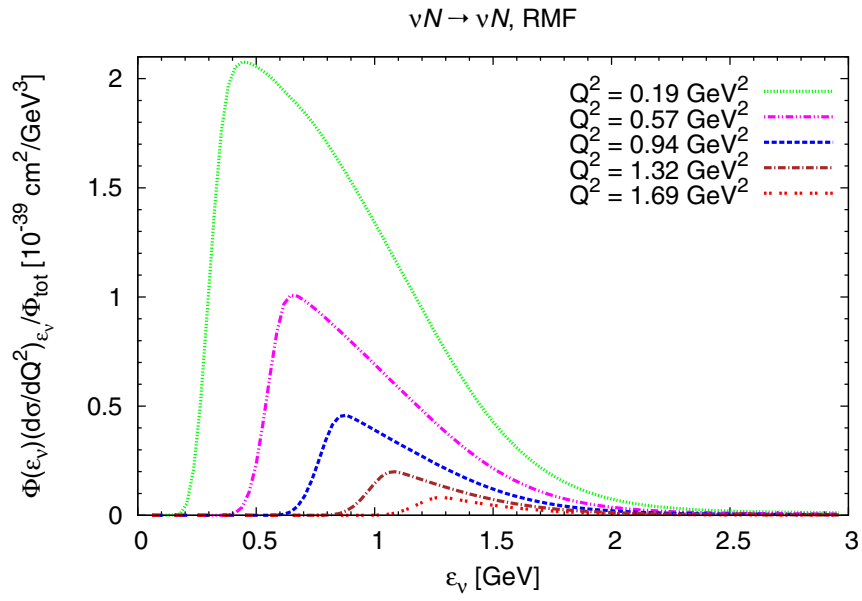

FIG. 4. (Color online) Differential cross section $d \sigma / d Q^{2}$ for $\nu$ NCQE scattering per bound nucleon in the ${ }^{12} \mathrm{C}$ nucleus calculated within the RMF model and multiplied by the MiniBooNE flux. Results are given at several fixed values of four-momentum transfer squared $\left(Q^{2}\right)$.

neutrons (per bound neutron). As shown in Fig. 3 the theoretical results corresponding to all models except the RGF-DEM underestimate the neutrino data in the region $0.1<Q^{2}<0.7 \mathrm{GeV}^{2}$, while all theories are within the error bars for higher $Q^{2}$. On the other hand, the same models underestimate the antineutrino data at high $Q^{2}$. This is clearly shown in the insets in Fig. 3, where the cross sections are represented on a logarithmic scale. The RGF-DEM results are larger than the results of the other models and in generally good agreement with the data over the entire $Q^{2}$ region considered in the figure. The enhancement of the RGF cross sections is due to the contribution of final-state channels that are recovered by the imaginary part of the optical potential and that are not included in the other models.

In order to better understand the behavior of our results with $Q^{2}$, we present in Fig. 4 the differential cross sections $d \sigma / d Q^{2}$ for $\nu$ NCQE scattering (per bound nucleon in the ${ }^{12} \mathrm{C}$ nucleus) calculated within the RMF model and multiplied by the MiniBooNE flux, at several fixed values of $Q^{2}$. $\Phi_{\text {tot }}$ is the total integrated $v_{\mu}$ flux factor for the MiniBooNE experiment:

$$
\Phi_{\mathrm{tot}}=\int \Phi\left(\varepsilon_{v}\right) d \varepsilon_{v}
$$

As shown in Fig. 4 for small values of $Q^{2}$ the contribution to the cross sections is accumulated from a large neutrino energy range (for example, at $Q^{2}=0.19 \mathrm{GeV}^{2}$ the contributions to the cross section come from neutrino energies of from 0.2 to $2.5 \mathrm{GeV}$ ), whereas for higher $Q^{2}$ values, the energy range which makes a contribution to the cross section becomes smaller (for example, at $Q^{2}=1.69 \mathrm{GeV}^{2}$ contributions to the cross section come from neutrino energies of from 1.1 to $2.5 \mathrm{GeV}$ ). This suggests that the discrepancy between the RMF result and the experimental data at low $Q^{2}$ can be ascribed to the failure of the impulse approximation for low (anti)neutrino energies. For the other models (except the RGF) the situation is different for $v$ and $\bar{v}$ scattering. To better 


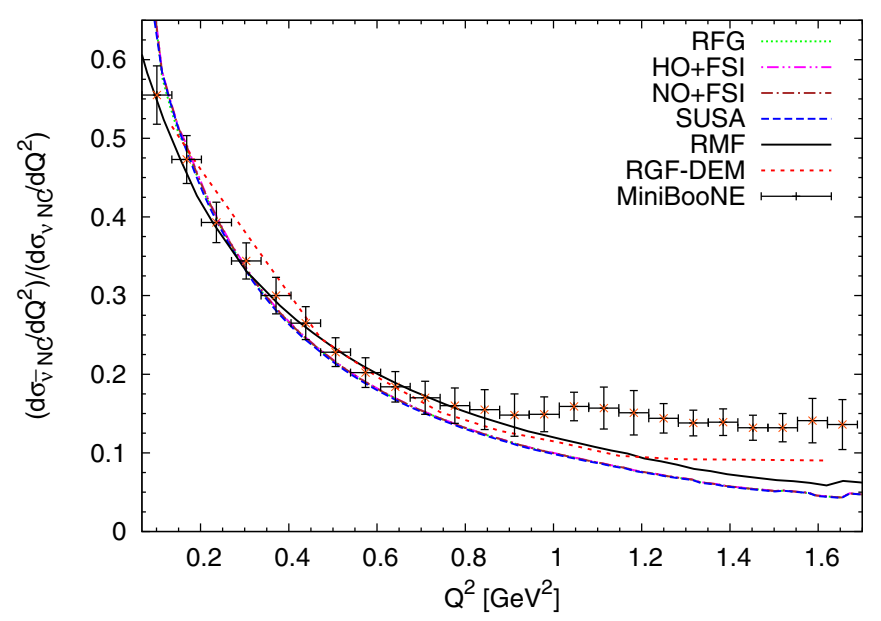

FIG. 5. (Color online) Ratio of the antineutrino-to-neutrino NCQE scattering cross section computed using the RFG, HO + FSI, $\mathrm{NO}+$ FSI, SUSA, RGF, and RMF approaches and compared with the MiniBooNE data [46].

illustrate this difference we show in Fig. 5 our predictions for the ratio of the antineutrino-to-neutrino NCQE scattering cross sections. The results are compared with the recently published MiniBooNE data [46]. Since both measurements were made in the same beamline and with the same detector, it is expected that the bin-to-bin ratio of the two cross-section measurements will cancel the common systematic errors. As shown in Fig. 5 the results of all models are in agreement with the data up to $Q^{2}<0.8 \mathrm{GeV}^{2}$ (actually the RMF and RGF results are in good agreement up to $Q^{2} \sim 1 \mathrm{GeV}^{2}$ ), whereas for bigger $Q^{2}$ all models underpredict the data. This is so because the theoretical antineutrino NCQE cross section underpredicts the data at high $Q^{2}$ (see Fig. 3). It must be noted that the results using different scaling functions almost coincide, since the cross sections are proportional to s.n. cross sections multiplied by the scaling function [see Eq. (1)] and the ratio is proportional to the ratio of the s.n. antineutrino-to-s.n. neutrino cross sections. The updated version of the SuSA model, SuSAv2, provides an interesting possibility in studies of NC cross sections and, particularly, of the mentioned ratio. This is related to the use of different parametrizations of the transverse and longitudinal scaling functions in SuSAv2. Work along this line is in progress.

For completeness we also present in Fig. 6 the spectra corresponding to the numerator and denominator entering the ratio between $v$ scattering from proton and nucleon (proton plus neutron) (left panels) and the ratio computed by dividing the two samples (right panel) within the various models and comparison with the MiniBooNE data [43]. The numerator and denominator data (left panels in Fig. 6) are taken from [90], where the data are reported without the corresponding
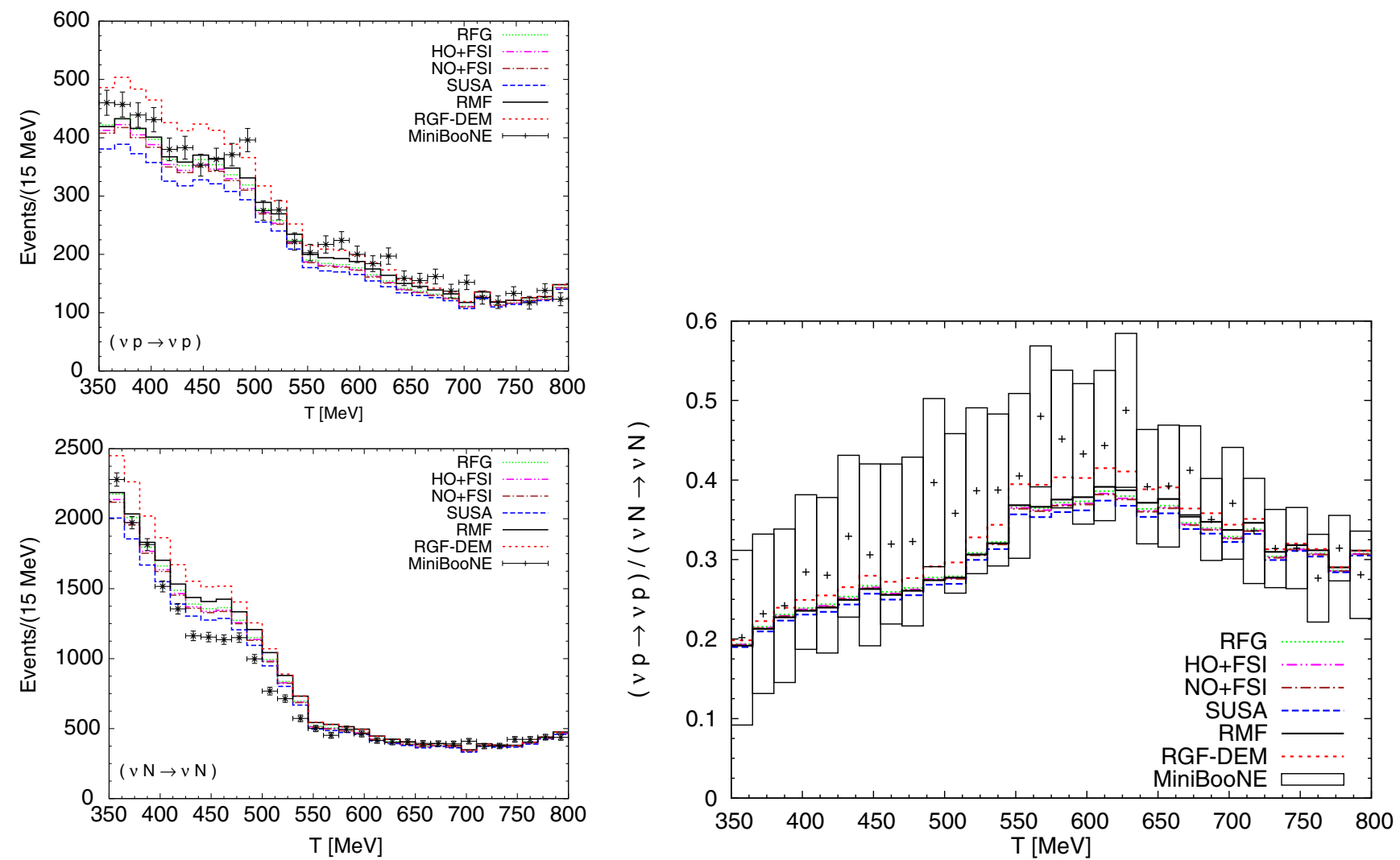

FIG. 6. (Color online) RFG, HO + FSI, NO + FSI, SUSA, RGF, and RMF predictions, after the folding procedure, compared with the histograms of the numerator (top left) and denominator (bottom left) of the ratio. Error bars in the left panel represent only the statistical uncertainty, computed as the square root of the event number. The corresponding ratio is shown in the right panel. The axial mass and strangeness are assumed to be the standard axial mass value and zero strangeness. Data are taken from $[43,89,90]$. 

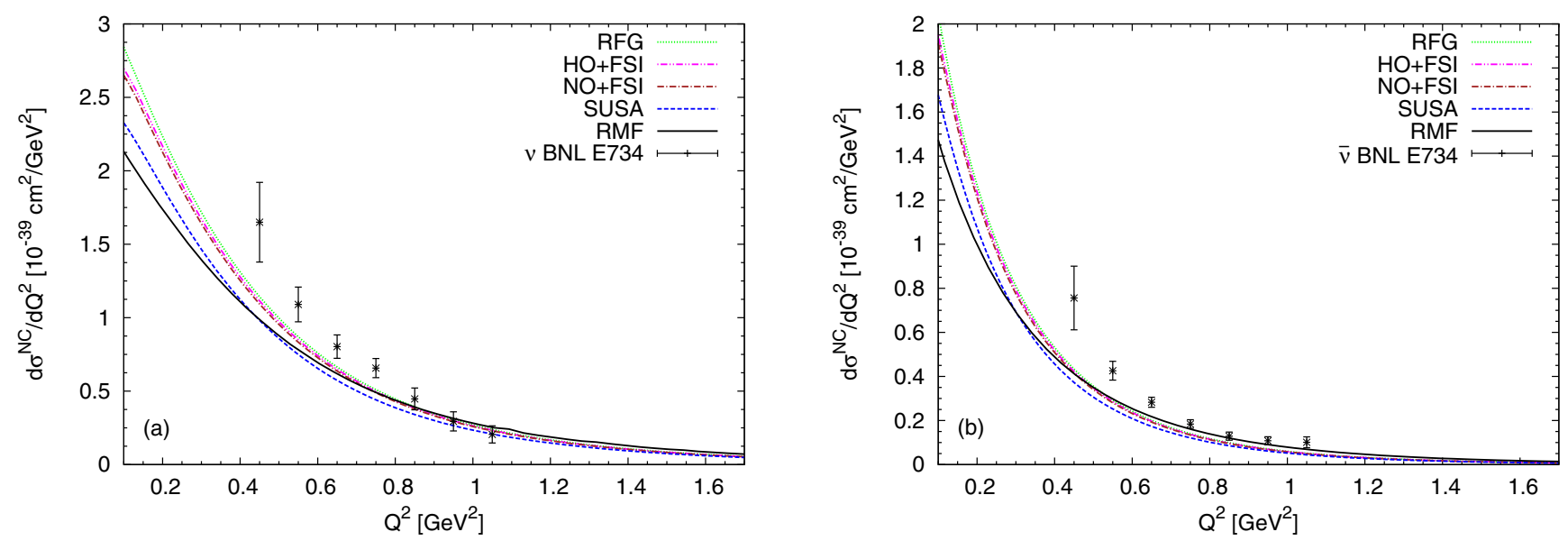

FIG. 7. (Color online) NCQE flux-averaged cross section: (a) $v p \rightarrow v p$ and (b) $\bar{v} p \rightarrow \bar{v} p$ compared with the BNL E734 experimental data [44]. Our results are evaluated using the RFG, HO + FSI, NO + FSI, and SUSA scaling functions in the RMF model with the standard value of the axial-vector dipole mass $M_{A}=1.032 \mathrm{GeV}^{2}$ and strangeness $\Delta s=0$. Error bars do not include the normalization uncertainty of $11.2 \%$ $(10.4 \%)$ in the $v(\bar{v})$ case.

errors (so in the figure, only statistical errors are included). In the calculations the axial mass and strangeness have been assumed to be the standard axial mass and zero strangeness. We note that the dispersion between the models tends to cancel when this ratio is considered. This result clearly shows that the proton/nucleon ratio is very insensitive to nuclear model effects and to FSIs, and hence, it may provide information that improves our present knowledge on the electroweak nucleon structure, in particular, the nucleon's strangeness. In particular, for kinetic energies of the outgoing nucleon $T_{N}>350 \mathrm{MeV}$ our models give results which are in good agreement with the experimental data (left panels in Fig. 6), while for the ratio our theoretical results are within the error bars at all kinematics (right panel in Fig. 6).

We now compare the results obtained with our models with the BNL E734 experimental data. The mean value of the neutrino (antineutrino) energy is $1.3 \mathrm{GeV}(1.2 \mathrm{GeV})$ for the BNL experiment, while for the MiniBooNE experiment it is $788 \mathrm{MeV}(665 \mathrm{MeV})$. In Fig. 7 the differential cross sections evaluated using the RFG, HO + FSI, NO + FSI, and SUSA scaling functions and the RMF model are compared with NCQE $v p \rightarrow v p$ [Fig. 7(a)] and $\bar{v} p \rightarrow \bar{v} p$ [Fig. 7(b)] BNL E734 experimental data. The BNL E734 experiment was performed using a 170-metric-ton high-resolution target detector in a horn-focused (anti)neutrino at the BNL. The cross-section results show a behavior similar to that in the MiniBooNE experiment. The latter [using the Cherenkov detector filled with mineral oil $\left.\left(\mathrm{CH}_{2}\right)\right]$ is sensitive to both $v(\bar{v}) p$ and $v(\bar{v}) n$ NCQE scattering $[43,46]$. It has been known for some time (see, e.g., [74-76]) that the $\Delta s$ dependence of the NCQE neutrino-nucleon cross section is very mild. This results from a cancellation between the effect of $\Delta s$ on the proton and that on the neutron contributions, which are affected differently by the axial strangeness: by changing $\Delta s$ from 0 to a negative value the proton cross section gets enhanced while the neutron one is reduced, so that the net effect on the total cross section is very small. NCQE $v(\bar{v}) p$ differential cross sections were measured in the BNL E734 experiment, which are sensitive to the values of $\Delta s$ (there is not a cancellation effect). The BNL
E734 experimental data can be reproduced within our models, in principle, by the fit of the axial strangeness without a change in the axial mass value.

Here we would like to mention, first, that our calculations using NO and $\mathrm{HO}$ single-particle wave functions in $n_{i}(p)$ in the spectral function, Eq. (8), with FSIs and without FSIs show that the inclusion of FSI effects leads to a small change (a depletion) in the cross sections. Second, the results for the cross sections obtained using the realistic spectral function $S(p, E)$ with single-particle momentum distributions $n_{i}(p)$ [see Eq. (8)] that include Jastrow short-range NN correlations (accounted for in the NOs) can be compared in Figs. 3, 5, 6, and 7 with those that do not include NN correlations (RFG and $\mathrm{HO}$ ). It can be seen that, similarly to the case of CCQE neutrino scattering (see Ref. [73]), the differences between results in correlated and results in noncorrelated approaches are small, thus showing that the process is not too sensitive to the specific treatment of the bound state.

\section{CONCLUSIONS}

This work complements previous studies focused on CCQE neutrino-nucleus scattering processes that were performed making use of a realistic spectral function. Here we extend this analysis to the case of NC neutrino processes and compare our theoretical predictions with data measured by the MiniBooNe and BNL collaborations. Contrary to CCQE reactions, where the final lepton is detected, in the NC case one has no information on the energy and momentum of the ejected neutrino. Hence the transferred 4-momentum cannot be determined. This, as discussed in some previous works, makes the description of the reaction mechanism not very clear and some caution should also be drawn regarding the "validity" of scaling arguments when applied to NC. However, our previous studies give us confidence in the reliability of our calculations and their application to describe the present experimental data measured at different facilities. 
The main objective of this work centers on the use of a realistic spectral function, which accounts for short-range NN correlations and also has a realistic energy dependence. This function gives a scaling function in accordance with electron scattering data and it can be used for a wide range of neutrino energies. Therefore, the use of this spectral function to describe the general reaction mechanism involved in NC neutrino-nucleus scattering processes can provide very valuable information that can be compared with results obtained with other theoretical approaches. In this sense, we compare our spectral function-based predictions with the results provided by the SuSA, RMF, and RGF models largely used by us in the past. The discrepancies found can help to disentangle effects directly linked to particular ingredients in the process: FSIs, nucleon correlations, effects beyond the impulse approximation, etc.

The predictions of our model agree in general with previous results, although with some peculiarities that should be emphasized. Our calculations show that the inclusion of FSI effects in the spectral-function-based calculations leads to a slight depletion of the cross section being in close agreement with the RFG prediction. The inclusion of FSI effects in the RGF model leads to larger cross sections, in good agreement with the data. On the contrary, the SuSA and, in particular, the RMF approaches lead to significantly smaller differential cross sections at low values of $\left|Q^{2}\right|\left(\leqslant 0.6-0.8 \mathrm{GeV}^{2}\right)$, also departing from the data. This behavior can be seen for the two experiments considered in this work: MiniBooNe and BNL. Another point of relevance when comparing the different models is the softer $Q^{2}$ dependence (with a smaller slope) shown by the RMF cross section. Whereas it is clearly below the other curves at low $Q^{2}$ (up to $\approx 0.5-0.6 \mathrm{GeV}^{2}$ ), it crosses them, providing the largest contribution, at higher $Q^{2}$. This result can be taken as an indication of the particular sensitivity of NC processes to the specific description of FSI effects. It may also be connected with the increasing tail in the scaling function provided by the RMF model at larger $Q^{2}$ values. This is a consequence of the enhancement of the lower components in the relativistic nucleon wave functions, particularly for the final state.
All our calculations are based on the impulse approximation, i.e., they do not include effects beyond the one-body approach, for example, $2 p-2 h$ contributions induced by MEC. These ingredients have been shown to be very important in the analysis of neutrino-nucleus scattering processes. In particular, they produce a significant enhancement in the cross section at low to moderate values of the transferred 4-momentum. This is consistent with our predictions that clearly underestimate data for such kinematical regions. On the contrary, the accordance improves at higher $Q^{2}$. This is strictly true for neutrinos at MiniBooNE. In the case of antineutrinos, MiniBooNE data at $Q^{2} \geqslant 1-1.2 \mathrm{GeV}^{2}$ are higher than theoretical predictions, the RMF results being closer to the experiment. This behavior also leads to the significant discrepancy observed for the antineutrino/neutrino ratio (Fig. 5). More studies are needed in order to understand these differences at medium to large $Q^{2}$ values. This could be related to a different role played by $2 p$ - $2 h$ contributions and MEC for neutrinos and antineutrinos. Work is in progress to evaluate the impact of $2 p$ - $2 h$ excitations on these results.

\section{ACKNOWLEDGMENTS}

This work was partially supported by Spanish DGI and FEDER funds (Grant Nos. FIS2011-28738-C02-01 and FPA2013-41267), by the Junta de Andalucia, by the Spanish Consolider-Ingenio 2000 program CPAN (Grant No. CSD2007-00042), by the Campus of Excellence International (CEI) of the Moncloa project (Madrid) and Andalucia Tech, and by the Istituto Nazionale di Fisica Nucleare under Contract MANYBODY, as well as by the Bulgarian National Science Fund under Contract Nos. DFNI-T02/19 and DFNIE02/6. M.V.I. is grateful for the warm hospitality given by the UCM and for financial support during his stay there from the SiNuRSE action within the ENSAR European project. A.N.A. acknowledges financial support from the Universidad de Sevilla under the Program "IV Plan Propio de Investigación. Movilidad de Investigadores." R.G.J. acknowledges financial help from VPPI-US (Universidad de Sevilla).
[1] G. B. West, Phys. Rep. 18, 263 (1975).

[2] I. Sick, D. B. Day, and J. S. McCarthy, Phys. Rev. Lett. 45, 871 (1980).

[3] C. Ciofi degli Atti, E. Pace, and G. Salmé, Phys. Rev. C 36, 1208 (1987).

[4] D. B. Day, J. S. McCarthy, T. W. Donnelly, and I. Sick, Annu. Rev. Nucl. Part. Sci. 40, 357 (1990).

[5] C. Ciofi degli Atti, E. Pace, and G. Salmé, Phys. Rev. C 43, 1155 (1991).

[6] C. Ciofi degli Atti and S. Simula, Phys. Rev. C 53, 1689 (1996).

[7] C. Ciofi degli Atti and G. B. West, arXiv:nucl-th/9702009.

[8] C. Ciofi degli Atti and G. B. West, Phys. Lett. B 458, 447 (1999).

[9] D. Faralli, C. Ciofi degli Atti, and G. B. West, in Proceedings of 2nd International Conference on Perspectives in Hadronic Physics, ICTP, Trieste, Italy, 1999, edited by S. Boffi, C. Ciofi degli Atti, and M. M. Giannini (World Scientific, Singapore, 2000), p. 75.

[10] W. M. Alberico, A. Molinari, T. W. Donnelly, E. L. Kronenberg, and J. W. Van Orden, Phys. Rev. C 38, 1801 (1988).

[11] M. B. Barbaro, R. Cenni, A. De Pace, T. W. Donnelly, and A. Molinari, Nucl. Phys. A 643, 137 (1998).

[12] T. W. Donnelly and I. Sick, Phys. Rev. Lett. 82, 3212 (1999).

[13] T. W. Donnelly and I. Sick, Phys. Rev. C 60, 065502 (1999).

[14] C. Maieron, T. W. Donnelly, and I. Sick, Phys. Rev. C 65, 025502 (2002).

[15] M. B. Barbaro, J. A. Caballero, T. W. Donnelly, and C. Maieron, Phys. Rev. C 69, 035502 (2004).

[16] A. N. Antonov, M. K. Gaidarov, D. N. Kadrev, M. V. Ivanov, E. Moya de Guerra, and J. M. Udias, Phys. Rev. C 69, 044321 (2004). 
[17] A. N. Antonov, M. K. Gaidarov, M. V. Ivanov, D. N. Kadrev, E. Moya de Guerra, P. Sarriguren, and J. M. Udías, Phys. Rev. C 71, 014317 (2005).

[18] A. N. Antonov, M. V. Ivanov, M. K. Gaidarov, E. Moya de Guerra, P. Sarriguren, and J. M. Udías, Phys. Rev. C 73, 047302 (2006).

[19] A. N. Antonov, M. V. Ivanov, M. K. Gaidarov, E. Moya de Guerra, J. A. Caballero, M. B. Barbaro, J. M. Udías, and P. Sarriguren, Phys. Rev. C 74, 054603 (2006).

[20] A. N. Antonov, M. V. Ivanov, M. K. Gaidarov, and E. Moya de Guerra, Phys. Rev. C 75, 034319 (2007).

[21] O. Benhar, D. Day, and I. Sick, Rev. Mod. Phys. 80, 189 (2008).

[22] Y. Fukuda et al. (The Super-Kamiokande Collaboration), Phys. Rev. Lett. 81, 1562 (1998); M. H. Ahn et al. (K2K. Collaboration), ibid. 90, 041801 (2003); Q.-R. Ahmad et al. (SNO Collaboration), ibid. 87, 071301 (2001); 89, 011301 (2002); K. Eguchi et al. (KamLAND Collaboration), ibid. 90, 021802 (2003); C. Athanassopoulos et al. (LSND Collaboration), ibid. 77, 3082 (1996); C. Athanassopoulos et al. (SNO Collaboration), ibid. 81, 1774 (1998).

[23] A. N. Antonov, V. A. Nikolaev, and I. Zh. Petkov, Bulg. J. Phys. 6, 151 (1979); Z. Phys. A 297, 257 (1980); 304, 239 (1982); Nuovo Cimento A 86, 23 (1985); 102, 1701 (1989); A. N. Antonov, D. N. Kadrev, and P. E. Hodgson, Phys. Rev. C 50, 164 (1994).

[24] A. N. Antonov, P. E. Hodgson, and I. Zh. Petkov, Nucleon Momentum and Density Distributions in Nuclei (Clarendon Press, Oxford, 1988); Nucleon Correlations in Nuclei (SpringerVerlag, Berlin, 1993).

[25] A. N. Antonov, M. V. Ivanov, M. B. Barbaro, J. A. Caballero, E. Moya de Guerra, and M. K. Gaidarov, Phys. Rev. C 75, 064617 (2007).

[26] J. E. Amaro, M. B. Barbaro, J. A. Caballero, T. W. Donnelly, A. Molinari, and I. Sick, Phys. Rev. C 71, 015501 (2005).

[27] J. A. Caballero, J. E. Amaro, M. B. Barbaro, T. W. Donnelly, C. Maieron, and J. M. Udías, Phys. Rev. Lett. 95, 252502 (2005).

[28] J. A. Caballero, Phys. Rev. C 74, 015502 (2006).

[29] J. E. Amaro, M. B. Barbaro, J. A. Caballero, T. W. Donnelly, and C. Maieron, Phys. Rev. C 71, 065501 (2005).

[30] J. E. Amaro, M. B. Barbaro, J. A. Caballero, and T. W. Donnelly, Phys. Rev. C 73, 035503 (2006).

[31] A. Meucci, C. Giusti, and F. D. Pacati, Nucl. Phys. A 744, 307 (2004).

[32] M. B. Barbaro, Nucl. Phys. B Proc. Suppl. 159, 186 (2006).

[33] M. C. Martínez, P. Lava, N. Jachowicz, J. Ryckebusch, K. Vantournhout, and J. M. Udías, Phys. Rev. C 73, 024607 (2006).

[34] J. Nieves, M. Valverde, and M. J. Vicente-Vacas, Nucl. Phys. B Proc. Suppl. 155, 263 (2006).

[35] M. B. Barbaro, J. E. Amaro, J. A. Caballero, T. W. Donnelly, and A. Molinari, Nucl. Phys. B Proc. Suppl. 155, 257 (2006).

[36] C. Maieron, M. C. Martinez, J. A. Caballero, and J. M. Udías, Phys. Rev. C 68, 048501 (2003).

[37] A. Meucci, C. Giusti, and F. D. Pacati, Nucl. Phys. A 739, 277 (2004); 773, 250 (2006).

[38] O. Benhar, Nucl. Phys. B Proc. Suppl. 139, 15 (2005); O. Benhar and N. Farina, ibid. 139, 230 (2005); O. Benhar, N. Farina, H. Nakamura, M. Sakuda, and R. Seki, ibid. 155, 254 (2006); Phys. Rev. D 72, 053005 (2005).

[39] G. Co', Nucl. Phys. B Proc. Suppl. 159, 192 (2006); A. Botrugno and G. Co', Nucl. Phys. A 761, 200 (2005); M. Martini, G. Co',
M. Anguiano, and A. M. Lallena, Phys. Rev. C 75, 034604 (2007).

[40] T. Leitner, L. Alvarez-Ruso, and U. Mosel, Phys. Rev. C 73, 065502 (2006).

[41] B. Szczerbinska, T. Sato, K. Kubodera, and T.-S. H. Lee, Phys. Lett. B 649, 132 (2007).

[42] A. A. Aguilar-Arevalo et al. (MiniBooNE Collaboration), Phys. Rev. D 81, 092005 (2010).

[43] A. A. Aguilar-Arevalo et al. (MiniBooNE Collaboration), Phys. Rev. D 82, 092005 (2010).

[44] K. Abe et al., Phys. Rev. Lett. 56, 1107 (1986); 56, 1883(E) (1986); L. A. Ahrens et al., Phys. Rev. D 35, 785 (1987).

[45] A. A. Aguilar-Arevalo et al. (MiniBooNE Collaboration), Phys. Rev. D 88, 032001 (2013).

[46] A. A. Aguilar-Arevalo et al. (MiniBooNE Collaboration), Phys. Rev. D 91, 012004 (2015).

[47] A. M. Ankowski, Phys. Rev. C 86, 024616 (2012).

[48] P. Lipari, Nucl. Phys. B Proc. Suppl. 112, 274 (2002); G. P. Zeller, arXiv:hep-ex/0312061.

[49] A. Meucci, C. Giusti, and F. D. Pacati, Phys. Rev. D 84, 113003 (2011).

[50] A. V. Butkevich and D. Perevalov, Phys. Rev. C 84, 015501 (2011).

[51] O. Benhar and G. Veneziano, Phys. Lett. B 702, 433 (2011).

[52] M. Martini, M. Ericson, and G. Chanfray, Phys. Rev. C 84, 055502 (2011).

[53] J. Nieves, I. R. Simo, and M. J. V. Vacas, Phys. Lett. B 707, 72 (2012).

[54] J. E. Amaro, M. B. Barbaro, J. A. Caballero, T. W. Donnelly, and J. M. Udías, Phys. Rev. D 84, 033004 (2011).

[55] A. Meucci, M. B. Barbaro, J. A. Caballero, C. Giusti, and J. M. Udías, Phys. Rev. Lett. 107, 172501 (2011).

[56] V. Bernard, L. Elouadrhiri, and Ulf-G. Meißner, J. Phys. G 28, R1 (2002).

[57] M. Martini, M. Ericson, G. Chanfray, and J. Marteau, Phys. Rev. C 80, 065501 (2009).

[58] M. Martini, M. Ericson, G. Chanfray, and J. Marteau, Phys. Rev. C 81, 045502 (2010).

[59] R. González-Jiménez, M. V. Ivanov, M. B. Barbaro, J. A. Caballero, and J. M. Udías, Phys. Lett. B 718, 1471 (2013)

[60] M. V. Ivanov, R. González-Jiménez, J. A. Caballero, M. B. Barbaro, T. W. Donnelly, and J. M. Udías, Phys. Lett. B 727, 265 (2013).

[61] R. González-Jiménez, J. A. Caballero, A. Meucci, C. Giusti, M. B. Barbaro, M. V. Ivanov, and J. M. Udías, Phys. Rev. C 88, 025502 (2013).

[62] A. Meucci and C. Giusti, Phys. Rev. D 89, 057302 (2014).

[63] A. Meucci, J. A. Caballero, C. Giusti, F. D. Pacati, and J. M. Udías, Phys. Rev. C 80, 024605 (2009).

[64] A. Meucci and C. Giusti, Phys. Rev. D 85, 093002 (2012).

[65] J. E. Amaro, M. B. Barbaro, J. A. Caballero, T. W. Donnelly, and C. F. Williamson, Phys. Lett. B 696, 151 (2011).

[66] R. González-Jiménez, G. D. Megias, M. B. Barbaro, J. A. Caballero, and T. W. Donnelly, Phys. Rev. C 90, 035501 (2014)

[67] O. Lalakulich, K. Gallmeister, and U. Mosel, Phys. Rev. C 86, 014614 (2012).

[68] V. Lyubushkin et al. (NOMAD Collaboration), Eur. Phys. J. C 63, 355 (2009). 
[69] T. Golan, Kr. M. Graczyk, C. Juszczak, and J. T. Sobczyk, Phys. Rev. C 88, 024612 (2013).

[70] A. Bodek, H. S. Budd, and M. E. Christie, Eur. Phys. J. C 71, 1726 (2011).

[71] J. T. Sobczyk, Eur. Phys. J. C 72, 1850 (2012).

[72] A. M. Ankowski and O. Benhar, Phys. Rev. D 88, 093004 (2013).

[73] M. V. Ivanov, A. N. Antonov, J. A. Caballero, G. D. Megias, M. B. Barbaro, E. Moya de Guerra, and J. M. Udías, Phys. Rev. C 89, 014607 (2014).

[74] M. B. Barbaro, A. De Pace, T. W. Donnelly, A. Molinari, and M. J. Musolf, Phys. Rev. C 54, 1954 (1996).

[75] W. M. Alberico, M. B. Barbaro, S. M. Bilenky, J. A. Caballero, C. Giunti, C. Maieron, E. Moya de Guerra, and J. M. Udías, Nucl. Phys. A 623, 471 (1997).

[76] W. M. Alberico, M. B. Barbaro, S. M. Bilenky, J. A. Caballero, C. Giunti, C. Maieron, E. Moya de Guerra, and J. M. Udías, Nucl. Phys. A 651, 277 (1999).

[77] M. C. Martinez, J. A. Caballero, T. W. Donnelly, and J. M. Udías, Phys. Rev. C 77, 064604 (2008).

[78] M. C. Martinez, J. A. Caballero, T. W. Donnelly, and J. M. Udías, Phys. Rev. Lett. 100, 052502 (2008).
[79] J. Liu, R. D. McKeown, and M. J. Ramsey-Musolf, Phys. Rev. C 76, 025202 (2007).

[80] R. Gonzalez-Jimenez, J. A. Caballero, and T. W. Donnelly, Phys. Rep. 524, 1 (2013).

[81] E. L. Lomon, Phys. Rev. C 66, 045501 (2002).

[82] E. L. Lomon, Phys. Rev. C 64, 035204 (2001).

[83] C. Crawford et al., Phys. Rev. C 82, 045211 (2010).

[84] E. D. Cooper, S. Hama, B. C. Clark, and R. L. Mercer, Phys. Rev. C 47, 297 (1993).

[85] F. Capuzzi, C. Giusti, and F. D. Pacati, Nucl. Phys. A 524, 681 (1991).

[86] A. Meucci, F. Capuzzi, C. Giusti, and F. D. Pacati, Phys. Rev. C 67, 054601 (2003).

[87] A. Meucci, J. A. Caballero, C. Giusti, and J. M. Udías, Phys. Rev. C 83, 064614 (2011).

[88] E. D. Cooper, S. Hama, and B. C. Clark, Phys. Rev. C 80, 034605 (2009).

[89] D. Perevalov, Neutrino-nucleus neutral current elastic interactions measurement in MiniBooNE, Ph.D. thesis, University of Alabama (2009).

[90] http://www-boone.fnal.gov/for_physicists/data_release/ncel 